\title{
Relationship between hours spent on the Internet and Web 2.0 in Higher Education
}

\section{Ángel Boza Carreño' and Sara Conde Vélez ${ }^{2}$}

1. University of Huelva (UHU), Spain | aboza@uhu.es

Submitted in: July 2014

2. University of Huelva (UHU), Spain | sara.conde@dedu.uhu.es

\section{Recommended citation}

Boza, A., \& Conde, S. (2015). Relationship between hours spent on the Internet and Web 2.0 in Higher Education. RUSC. Universities and Knowledge Society Journal, 12(3). pp. 86-97. doi: http://dx.doi.org/10.7238/rusc.v12i3.2280

\begin{abstract}
This article analyses the relationship between the number of hours spent by university students on the Internet and their attitude, training, use, impact and perception of difficulties in Web 2.0 integration, as well as their knowledge and use of Web 2.0 tools in Higher Education. To this end, we used a Likert scale applied to a sample of 403 students from the University of Huelva (UHU), Spain. To test the hypothesis, we conducted a one-way ANOVA with post hoc comparisons. The results obtained partially confirmed the general hypothesis. The greatest differences were found in the factors of impact and use of Web 2.0 tools, whereas the least differences were found in the factors of attitude, training, difficulties and knowledge of Web 2.0 tools.
\end{abstract}

\section{Keywords}

ICTs, Internet, Web 2.0, training, attitude, use, impact, university education

\section{Relación entre horas dedicadas a internet y web 2.0 en educación universitaria}

\section{Resumen}

El presente artículo analiza la relación existente entre el número de horas diarias que el alumnado universitario dedica a internet y la actitud, formación, uso, impacto, percepción de dificultades de la integración de la web 2.0, así como el conocimiento y uso de herramientas de la web 2.0 en educación universitaria. Para ello hemos usado una escala tipo Likert aplicada a una muestra de 403 alumnos de la Universidad de Huelva. Para contrastar la hipótesis realizamos una ANOVA de un factor con comparaciones post hoc. Los resultados obtenidos permiten confirmar parcialmente la hipótesis general. Las diferencias mayores se observan en los factores de impacto y uso de herramientas de la web 2.0, mientras que las diferencias menores se observan en los factores de actitud, formación, dificultades y conocimiento de herramientas de la web 2.0.

\section{Palabras clave}

TIC, internet, web 2.0, formación, actitud, uso, impacto, educación universitaria

RUSC VOL. 12 No 3 | Universitat Oberta de Catalunya and University of New England | Barcelona, July 2015 


\section{Introduction}

In recent years, there has been a dramatic increase in the utilisation of information and communication technologies (ICTs). The use of the Internet in particular has become normalised as part of everyday human activity. An increasing number of people now use the Internet to engage in activities which until recently took place in real life (Casas, Ruiz-Olivares, \& Ortega-Ruiz, 2012). There is currently no conception of learning that does not include ICTs and the Internet. Another step in this development of connectivity is Web 2.0, which enables us to develop new virtual education based on tools for common creation, distributed authoring and virtual interaction in networks (Aguirre \& Manasía, 2009, p. 321).

Many research works have focused on studying this phenomenon, highlighting a series of factors which appear to be relevant to Web 2.0 integration in education: training is one of the main obstacles hindering the use of ICTs in education, as the training of teachers and students is considered essential (Hinojo \& Fernández, 2002; Ruiz, Rubia, Martínez, \& Fernández, 2010). Training in ICTs and their use by university teaching staff has been the subject of research and reflection in recent years (Alba, 2005; Alba \& Carballo, 2005; Area, 2004; Cabero, 2014; Sánchez \& Mayor, 2006; Tejedor, García, \& Prada, 2009).

The second factor is the attitudes towards ICTs. Most studies analyse the differences between attitudes towards new technological resources and their use (Boza, Tirado, \& Guzmán, 2010; Cavas-Bulent, Cavas-Pinar, Karaoglan, \& Kisla, 2009; Martínez, Amenabar, \& Lareki, 2011).

The third factor is the use of ICTs. Research highlights their usefulness as a space for storage and dissemination of documents, evidencing the application of a pedagogical model that does not significantly add anything to the technological systems, as virtual educational uses are still approached with traditional parameters (Suárez, 2009). But they can also become a new educational scenario, characterised by a virtual representation of the teaching/ learning process and a restructuring of the usual way of working in education (Díez, 2012). Pérez Ríos (2003) or Sangrà and González (2004) considered the use of ICTs in Higher Education as an appropriate means to improve the educational quality of their programmes.

The fourth factor is the impact of Web 2.0 on teaching-learning processes, described in the works of Redecker, Ala-Mutka, Bacigahpo, Ferrari, and Punie (2009) and Dabbagh and Reo (2011). They emphasise the use of Web 2.0 as a dominant force for action in Higher Education that promotes significant changes, providing new tools and novel, more cost-effective formats for knowledge, as well as personalised learning experiences. However, the way an institution incorporates Web 2.0 into existing processes will determine the positive impact of this innovation.

The fifth and last factor is the difficulties or challenges faced in Web 2.0 integration, mainly highlighting lack of confidence, lack of competence and negative attitudes towards change (Albirini, 2006; Ertmer, 2005). The British Educational Communications and Technology Agency (BECTA, 2004) points out the resistance to change and the negative attitude held regarding the possible use of these technologies to improve teaching and learning. Different studies also underscore the lack of time (BECTA, 2004; Osborne \& Hennessy, 2003), lack of training (Cuadrado, 2008; Toprakci, 2006; Valdes, Angulo, Urías, García, \& Mortis, 2011; Ballesteros, Cabero, Llorente, \& Morales, 2010) and the difficulties of access (BECTA, 2004).

Finally, a review of research on Web 2.0 tools used in teaching-learning activities highlighted the role of wikis, blogs, forums, podcasts, Moodle and Webquest as aids in collaborative learning, as well as in change and innovation (Garay, Luján, \& Etxebarría, 2013; Díez, 2012).

RUSC VOL. 12 No 3 | Universitat Oberta de Catalunya and University of New England | Barcelona, July 2015

@Ángel Boza Carreño and Sara Conde Vélez @ @ by FUOC, 2015 | Relationship between hours spent on the Internet and Web 2.0 in Higher Education 
However, the scarcity of studies linking the hours spent on the Internet and Web 2.0 in university students justifies the interest of this research, whose initial hypothesis is that students that spend more time on the Internet are positively differentiated from those who spend fewer hours in terms of their attitude, training, impact, perception of difficulties, knowledge and use of Web 2.0 in education.

\section{Method}

\section{Participants}

The population under study is taking degree courses in Educational Psychology, Psychology, Social Education, Early Childhood Education, Primary Education, Music Education, Special Education, Foreign Language Teaching, Industrial Relations and Nursing at the University of Huelva (UHU), Spain. The random cluster sample used ensures adequate representation and consists of 403 students, with a confidence level of $95.5 \%$ and estimated sampling error of 4.8\%, of which 172 are men and 231 are women, with a mean age of 23.02 years and an average of 3.96 hours spent on the Internet daily.

\section{Instrument and variables}

For data collection, a Likert scale with values from 1 to 7 was used, consisting of the following factors: attitude, training, use, impact and difficulties of Web 2.0 in Higher Education, knowledge and use of Web 2.0 tools, which had been previously validated (Boza \& Conde, 2015).

\section{Data analysis}

To test the hypothesis, we carried out an ANOVA 1×3. To this end, we proceeded to categorise the variable daily hours of Internet use into three user groups based on the mean (3.96) plus/minus a standard deviation (2.6), resulting in a low-use group (1-2 hours), a medium-use group (3-6 hours) and a high-use group (7 or more hours). Next, post hoc comparisons were drawn using the Bonferroni or Tamhane tests based on assumed equal variances or not, respectively, analysed by Levene's test.

\section{Results}

Below we present a breakdown of the results by factor, first noting the sub-hypothesis to be tested:

- H1: Students who spend more hours on the Internet are positively differentiated from those who spend fewer hours in their attitude towards Web 2.0 integration in education. 
Table 1. Attitude towards Web 2.0 and hours of Internet use $\left({ }^{*}=p<0.05 ;{ }^{* *}=p<0.01 ; *^{* *}=p<0.001\right)$

\begin{tabular}{|c|c|c|c|c|c|c|c|c|c|c|c|}
\hline \multirow{3}{*}{$\frac{8}{8}$} & \multirow{3}{*}{ ITEM } & \multicolumn{6}{|c|}{ INTERNET HOURS } & \multirow{3}{*}{$\begin{array}{c}\text { ANOVA } \\
F\end{array}$} & \multicolumn{3}{|c|}{ MEAN DIFFERENCES } \\
\hline & & \multicolumn{2}{|c|}{$\begin{array}{c}1-2 h \text { (low) } \\
N=113\end{array}$} & \multicolumn{2}{|c|}{$\begin{array}{c}\text { 3-6 } h \text { (medium) } \\
N=212\end{array}$} & \multicolumn{2}{|c|}{$\begin{array}{c}7 \text { or more h (high) } \\
\qquad N=78\end{array}$} & & \multirow{2}{*}{$\begin{array}{l}\text { Medium/ } \\
\text { low }\end{array}$} & \multirow{2}{*}{$\begin{array}{l}\text { High/ } \\
\text { low }\end{array}$} & \multirow{2}{*}{$\begin{array}{l}\text { High/ } \\
\text { medium }\end{array}$} \\
\hline & & $M$ & SD & $M$ & SD & $M$ & SD & & & & \\
\hline \multirow{8}{*}{ 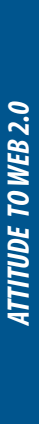 } & A5. Tool for quality education & 5.17 & 1.47 & 5.17 & 1.18 & 4.69 & 1.79 & $3.63^{*}$ & 0.00 & -0.47 & -0.47 \\
\hline & A6. A passing fad & 2.95 & 1.88 & 3.13 & 1.62 & 2.82 & 1.43 & 1.10 & 0.18 & -0.12 & -0.30 \\
\hline & A7. Younger teachers are more predisposed & 5.65 & 5.90 & 5.30 & 1.32 & 5.44 & 1.32 & 0.40 & -0.34 & -0.21 & 0.13 \\
\hline & A8. Attractive and novel for students & 5.52 & 1.13 & 5.32 & 1.19 & 5.21 & 1.48 & 1.67 & -0.20 & -0.31 & -0.11 \\
\hline & A9. Easier work & 5.06 & 1.22 & 5.15 & 1.32 & 4.85 & 1.69 & 1.39 & 0.08 & -0.21 & -0.30 \\
\hline & $\begin{array}{l}\text { A10. Teachers reluctant due to lack of } \\
\text { knowledge }\end{array}$ & 5.14 & 1.52 & 4.93 & 1.41 & 4.87 & 1.55 & 0.99 & -0.20 & -0.27 & -0.06 \\
\hline & A11. Helps personalise education & 4.94 & 1.47 & 4.55 & 1.33 & 4.49 & 1.48 & $3.41^{*}$ & -0.38 & -0.45 & -0.06 \\
\hline & A12. Teachers' positive attitude towards use & 4.50 & 1.35 & 4.37 & 1.38 & 4.47 & 1.50 & 0.40 & -0.13 & -0.03 & 0.10 \\
\hline
\end{tabular}

Overall, the hypothesis was rejected. No significant differences were found in attitude towards Web 2.0 in any of the following indicators: tool for quality education; a passing fad; younger teachers are more predisposed; attractive and novel for students; easier work; teachers reluctant due to lack of knowledge; or teachers' positive attitude towards use. Significant differences were found in only two of the eight indicators evaluated. Students who use the Internet more were also more likely to view Web 2.0 as a tool for quality education. In contrast, those spending less time on the Internet were more likely to think that Web 2.0 helps personalise education.

- H2: Students who spend more hours on the Internet are positively differentiated from those who spend fewer hours browsing in their attitude towards training for Web 2.0 integration in education.

Table 2. Training in Web 2.0 and hours of Internet use $\left(^{*}=p<0.05 ;{ }^{* *}=p<0.01 ;{ }^{* * *}=p<0.001\right)$

\begin{tabular}{|c|c|c|c|c|c|c|c|c|c|c|c|}
\hline \multirow{3}{*}{ 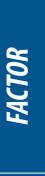 } & \multirow{3}{*}{ ITEM } & \multicolumn{6}{|c|}{ INTERNET HOURS } & \multirow{3}{*}{$\begin{array}{c}\text { ANOVA } \\
F\end{array}$} & \multicolumn{3}{|c|}{ MEAN DIFFERENCES } \\
\hline & & \multicolumn{2}{|c|}{$\begin{array}{c}1-2 h \text { (low) } \\
N=113\end{array}$} & \multicolumn{2}{|c|}{$\begin{array}{c}\text { 3-6 } h \text { (medium) } \\
N=212\end{array}$} & \multicolumn{2}{|c|}{$\begin{array}{c}7 \text { or more } h \text { (high) } \\
\qquad=78\end{array}$} & & \multirow{2}{*}{$\begin{array}{c}\text { Medium/ } \\
\text { low }\end{array}$} & \multirow{2}{*}{$\begin{array}{l}\text { High/ } \\
\text { low }\end{array}$} & \multirow{2}{*}{$\begin{array}{l}\text { High/ } \\
\text { medium }\end{array}$} \\
\hline & & $M$ & SD & $M$ & SD & $M$ & SD & & & & \\
\hline \multirow{9}{*}{ 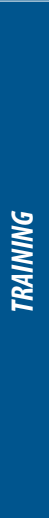 } & T13. Known by teachers & 4.34 & 1.51 & 4.21 & 1.58 & 3.79 & 1.66 & 2.90 & -0.12 & -0.54 & -0.41 \\
\hline & T14. Teachers are suitably trained & 3.77 & 1.54 & 3.69 & 1.46 & 3.71 & 1.34 & 0.10 & -0.07 & -0.06 & 0.01 \\
\hline & T15. Training more technical than didactic & 4.38 & 1.49 & 4.35 & 1.58 & 4.14 & 1.62 & 0.62 & -0.03 & -0.24 & -0.20 \\
\hline & $\begin{array}{l}\text { T16. Students have received sufficient } \\
\text { training }\end{array}$ & 3.76 & 1.54 & 3.66 & 1.49 & 3.49 & 1.68 & 0.72 & -0.10 & -0.27 & -0.16 \\
\hline & $\begin{array}{l}\text { T17. Teacher training comes from their } \\
\text { experiences }\end{array}$ & 4.58 & 1.67 & 4.43 & 1.49 & 4.64 & 1.83 & 0.60 & -0.15 & 0.05 & 0.20 \\
\hline & T18. A teacher training plan is necessary & 5.22 & 1.55 & 5.33 & 1.40 & 5.51 & 1.48 & 0.92 & 0.10 & 0.29 & 0.18 \\
\hline & T19. Lack of motivation in teaching & 4.72 & 1.68 & 4.98 & 1.41 & 5.37 & 2.55 & $3.18^{*}$ & 0.26 & $0.65^{*}$ & 0.39 \\
\hline & T20. Training courses are the way to learn & 4.74 & 1.53 & 4.78 & 1.36 & 4.35 & 1.71 & 2.57 & 0.04 & -0.39 & -0.43 \\
\hline & T21. Students have clear knowledge & 3.35 & 1.49 & 3.77 & 1.62 & 3.14 & 1.58 & $4.92^{* *}$ & 0.42 & -0.21 & $-0.63^{*}$ \\
\hline
\end{tabular}

Overall, the hypothesis was rejected. No significant differences were found in training in Web 2.0 in any of the following indicators: known by teachers; teachers are suitably trained; training more technical than didactic; students have received sufficient training; teacher training comes from their experiences; a teacher training plan is necessary; or training courses are the way to learn. The only differentiation was in students who spend more time 
on the Internet believing that there is a lack of motivation in teaching and that, in the medium-use group, students have clear knowledge.

- H3: Students who spend more time on the Internet differ in their assessment of the use of Web 2.0 in education compared to students who spend fewer hours online.

Table 3. Use of Web 2.0 and hours of Internet use $\left(^{*}=p<0.05 ;{ }^{* *}=p<0.01 ;{ }^{* *}=p<0.001\right)$

\begin{tabular}{|c|c|c|c|c|c|c|c|c|c|c|c|}
\hline \multirow{3}{*}{ 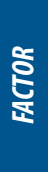 } & \multirow{3}{*}{ ITEM } & \multicolumn{6}{|c|}{ INTERNET HOURS } & \multirow{3}{*}{$\begin{array}{c}\text { ANOVA } \\
F\end{array}$} & \multicolumn{3}{|c|}{ MEAN DIFFERENCES } \\
\hline & & \multicolumn{2}{|c|}{$\begin{array}{c}1-2 h \text { (low) } \\
N=113\end{array}$} & \multicolumn{2}{|c|}{$\begin{array}{c}\text { 3-6 } h \text { (medium) } \\
N=212\end{array}$} & \multicolumn{2}{|c|}{$\begin{array}{c}7 \text { or more h (high) } \\
\qquad N=78\end{array}$} & & \multirow{2}{*}{$\begin{array}{l}\text { Medium/ } \\
\text { low }\end{array}$} & \multirow{2}{*}{$\begin{array}{l}\text { High/ } \\
\text { low }\end{array}$} & \multirow{2}{*}{$\begin{array}{l}\text { High/ } \\
\text { medium }\end{array}$} \\
\hline & & $M$ & SD & $M$ & SD & $M$ & SD & & & & \\
\hline \multirow{6}{*}{$\ddot{s}$} & $\begin{array}{l}\text { U22. Web } 2.0 \text { is limited to creation } \\
\text { of digital materials }\end{array}$ & 4.05 & 1.90 & 4.38 & 6.61 & 4.17 & 1.81 & 0.33 & 0.32 & 0.11 & -0.21 \\
\hline & U23. Complement to teaching & 4.68 & 1.75 & 4.74 & 1.19 & 5.01 & 1.45 & 1.41 & 0.05 & 0.33 & 0.27 \\
\hline & $\begin{array}{l}\text { U24. Teachers use Web } 2.0 \text { to post } \\
\text { information }\end{array}$ & 5.14 & 1.56 & 5.31 & 1.38 & 5.08 & 1.64 & 0.91 & 0.17 & -0.06 & -0.23 \\
\hline & $\begin{array}{l}\text { U25. Students handle Web } 2.0 \\
\text { easily }\end{array}$ & 4.58 & 1.52 & 4.74 & 1.49 & 4.77 & 1.61 & 0.48 & 0.15 & 0.18 & 0.02 \\
\hline & $\begin{array}{l}\text { U26. Teachers use Web } 2.0 \text { in their } \\
\text { teaching }\end{array}$ & 4.55 & 1.50 & 4.50 & 1.46 & 4.46 & 1.58 & 0.16 & -0.04 & -0.12 & -0.07 \\
\hline & $\begin{array}{l}\text { U27. Use of Web } 2.0 \text { has more } \\
\text { advantages than drawbacks }\end{array}$ & 5.27 & 1.42 & 5.04 & 1.26 & 4.83 & 1.70 & 2.35 & -0.23 & -0.44 & -0.20 \\
\hline
\end{tabular}

The hypothesis was rejected. No significant differences were found between them in any of the uses of Web 2.0 analysed: Web 2.0 is limited to creation of digital materials; complement to teaching; teachers use Web 2.0 to post information; students handle Web 2.0 easily; teachers use Web 2.0 in their teaching; or use of Web 2.0 has more advantages than drawbacks. 
- H4: Students who spend more time online estimate that Web 2.0 integration has greater impact than students who spend fewer hours on the Internet.

Table 4. Impact of Web 2.0 and hours of Internet use $\left(^{*}=p<0.05 ;^{* *}=p<0.01 ;^{* * *}=p<0.001\right)$

\begin{tabular}{|c|c|c|c|c|c|c|c|c|c|c|c|}
\hline \multirow{3}{*}{$\frac{c}{8}$} & \multirow{3}{*}{ ITEM } & \multicolumn{6}{|c|}{ INTERNET HOURS } & \multirow{3}{*}{$\begin{array}{c}\text { ANOVA } \\
F\end{array}$} & \multicolumn{3}{|c|}{ MEAN DIFFERENCES } \\
\hline & & \multicolumn{2}{|c|}{$\begin{array}{c}1-2 h(l o w) \\
N=113\end{array}$} & \multicolumn{2}{|c|}{$\begin{array}{c}\text { 3-6 } h \text { (medium) } \\
N=212\end{array}$} & \multicolumn{2}{|c|}{$\begin{array}{c}7 \text { or more h } \\
\text { (high) } \\
N=78\end{array}$} & & \multirow[t]{2}{*}{$\begin{array}{l}\text { Medium/ } \\
\text { low }\end{array}$} & \multirow[t]{2}{*}{$\begin{array}{l}\text { High/ } \\
\text { low }\end{array}$} & \multirow[t]{2}{*}{$\begin{array}{l}\text { High/ } \\
\text { medium }\end{array}$} \\
\hline & & $M$ & SD & $M$ & SD & $M$ & SD & & & & \\
\hline \multirow{15}{*}{ ז } & I28. Brings about change in teaching practice & 5.00 & 1.40 & 4.97 & 1.28 & 5.01 & 1.59 & 0.03 & -0.02 & 0.01 & 0.04 \\
\hline & 129. Major impact on social relations & 5.11 & 1.42 & 5.18 & 1.44 & 4.95 & 1.78 & 0.69 & 0.07 & -0.15 & -0.23 \\
\hline & I30. Favours collaborative learning experiences & 5.42 & 1.14 & 5.07 & 1.28 & 4.68 & 1.55 & $7.60^{* *}$ & $-0.35^{*}$ & $-0.74^{* *}$ & -0.39 \\
\hline & I31. Improves student-teacher communication & 4.99 & 1.51 & 4.94 & 1.35 & 4.26 & 1.61 & $7.40^{* *}$ & -0.04 & $-0.73^{*}$ & $-0.68^{*}$ \\
\hline & $\begin{array}{l}\text { 132. Students assume a more active role in } \\
\text { learning }\end{array}$ & 5.14 & 1.40 & 4.81 & 1.40 & 4.49 & 1.59 & $4.86^{* *}$ & -0.33 & $-0.65^{*}$ & -0.31 \\
\hline & $\begin{array}{l}\text { 133. Causes impoverishment of written } \\
\text { expression }\end{array}$ & 4.65 & 1.94 & 4.94 & 1.64 & 4.92 & 1.81 & 1.08 & 0.29 & 0.27 & -0.01 \\
\hline & 134. Improves academic performance & 4.24 & 1.39 & 4.32 & 1.36 & 4.04 & 1.40 & 1.19 & 0.082 & -0.20 & -0.28 \\
\hline & 135. Provides only superficial learning & 3.39 & 1.66 & 3.95 & 1.58 & 4.10 & 1.62 & $5.92^{* *}$ & $0.56^{*}$ & $0.71^{*}$ & 0.15 \\
\hline & 136. Improves interaction among teachers & 4.46 & 1.57 & 4.36 & 1.34 & 4.03 & 1.72 & 2.11 & -0.09 & -0.43 & -0.33 \\
\hline & I37. Improves subject methodology & 4.68 & 1.39 & 4.85 & 1.24 & 4.28 & 1.49 & $5.20^{* *}$ & -0.17 & $-0.57^{*}$ & -0.39 \\
\hline & 138. Generates more virtual tutorials & 4.86 & 1.69 & 4.89 & 1.40 & 4.29 & 1.83 & $4.29^{*}$ & 0.28 & -0.56 & $-0.59^{*}$ \\
\hline & $\begin{array}{l}\text { 139. Dehumanises the student-teacher } \\
\text { relationship }\end{array}$ & 3.58 & 1.75 & 3.98 & 1.63 & 4.28 & 1.78 & $4.20^{*}$ & 0.40 & $0.70^{*}$ & 0.30 \\
\hline & 140. Encourages student individuality & 3.84 & 1.67 & 4.17 & 1.60 & 4.44 & 1.53 & $3.29^{*}$ & 0.32 & $0.59^{*}$ & 0.26 \\
\hline & $\begin{array}{l}\text { 141. Foments autonomous student-centred } \\
\text { learning }\end{array}$ & 4.96 & 1.44 & 4.67 & 1.38 & 4.90 & 1.77 & 1.65 & -0.29 & -0.05 & 0.23 \\
\hline & 142. Generates greater commitment in students & 4.96 & 1.52 & 4.65 & 1.30 & 4.35 & 1.74 & $4.09^{*}$ & -0.31 & $-0.61^{*}$ & -0.30 \\
\hline
\end{tabular}

The data do not allow us to confirm or reject the hypothesis overall, but significant differences were found between some students and others. The analysis points to the perception of positive impacts by students that use the Internet less, and negative impacts by those using the Internet more. Students who use the Internet more are differentiated by believing that the impact of Web 2.0 provides only superficial learning; dehumanises the teacher-student relationship and encourages student individuality. In contrast, students who use the Internet less are distinguished by considering that the impact of Web 2.0 favours collaborative learning experiences; improves student-teacher communication; improves subject methodology; generates more virtual tutorials; students assume a more active role; and generates greater commitment in students. 
- H5: Students who spend more time online perceive more difficulties in Web 2.0 integration than students who spend fewer hours on the Internet.

Table 5. Hours of Internet use and difficulties with Web $2.0\left({ }^{*}=p<0.05 ;{ }^{* *}=p<0.01 ;{ }^{* *}=p<0.001\right)$

\begin{tabular}{|c|c|c|c|c|c|c|c|c|c|c|c|}
\hline \multirow{3}{*}{$\frac{\mathfrak{c}}{\mathrm{c}}$} & \multirow{3}{*}{ ITEM } & \multicolumn{6}{|c|}{ INTERNET HOURS } & ANOVA & \multicolumn{3}{|c|}{ MEAN DIFFERENCES } \\
\hline & & \multicolumn{2}{|c|}{$\begin{array}{c}1-2 h \text { (low) } \\
N=113\end{array}$} & \multicolumn{2}{|c|}{$\begin{array}{c}\text { 3-6 } h \text { (medium) } \\
N=212\end{array}$} & \multicolumn{2}{|c|}{$\begin{array}{c}7 \text { or more } h \text { (high) } \\
\qquad N=78\end{array}$} & \multirow[t]{2}{*}{$\boldsymbol{F}$} & \multirow{2}{*}{$\begin{array}{c}\text { Medium/ } \\
\text { low }\end{array}$} & \multirow{2}{*}{$\begin{array}{l}\text { High/ } \\
\text { low }\end{array}$} & \multirow{2}{*}{$\begin{array}{c}\text { High/ } \\
\text { medium }\end{array}$} \\
\hline & & $M$ & SD & $M$ & SD & $M$ & SD & & & & \\
\hline \multirow{3}{*}{ 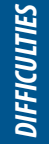 } & D43. Teachers find it hard to adapt & 4.58 & 1.64 & 4.48 & 1.58 & 4.91 & 1.45 & 2.15 & -0.10 & 0.32 & 0.43 \\
\hline & D44. An extra workload for teachers & 4.43 & 1.77 & 4.32 & 1.67 & 4.82 & 1.56 & 2.52 & -0.11 & 0.38 & 0.50 \\
\hline & D45. Technical issues a drawback & 5.42 & 1.54 & 5.00 & 1.57 & 5.63 & 1.35 & $5.90^{* *}$ & -0.41 & 0.21 & $0.62^{*}$ \\
\hline
\end{tabular}

The hypothesis was partially confirmed. Students who spend many hours on the Internet are differentiated by tending to consider technical issues a drawback. They are also more likely to perceive as difficulties that teachers find it hard to adapt and that Web 2.0 involves an extra workload for teachers, although these differences are not significant.

- H6: Students who spend more time online have more knowledge of Web 2.0 tools than students who spend fewer hours on the Internet.

Table 6. Hours of Internet use and knowledge of Web $2.0\left(^{*}=p<0.05 ;{ }^{* *}=p<0.01 ;{ }^{* *}=p<0.001\right)$

\begin{tabular}{|c|c|c|c|c|c|c|c|c|c|c|c|}
\hline \multirow{3}{*}{$\frac{\dddot{c}}{8}$} & \multirow{3}{*}{ ITEM } & \multicolumn{6}{|c|}{ INTERNET HOURS } & \multirow{3}{*}{$\begin{array}{c}\text { ANOVA } \\
F\end{array}$} & \multicolumn{3}{|c|}{ MEAN DIFFERENCES } \\
\hline & & \multicolumn{2}{|c|}{$\begin{array}{c}1-2 h(\text { low }) \\
N=113\end{array}$} & \multicolumn{2}{|c|}{$\begin{array}{c}3-6 h \text { (medium) } \\
N=212\end{array}$} & \multicolumn{2}{|c|}{$\begin{array}{c}7 \text { or more h (high) } \\
\qquad N=78\end{array}$} & & \multirow{2}{*}{$\begin{array}{c}\text { Medium/ } \\
\text { low }\end{array}$} & \multirow{2}{*}{$\begin{array}{l}\text { High/ } \\
\text { low }\end{array}$} & \multirow{2}{*}{$\begin{array}{l}\text { High/ } \\
\text { medium }\end{array}$} \\
\hline & & $M$ & SD & $M$ & SD & $M$ & SD & & & & \\
\hline \multirow{12}{*}{$\begin{array}{l}\frac{n}{8} \\
\frac{8}{8} \\
\frac{4}{6} \\
\frac{\mathbf{y}}{3} \\
\frac{3}{3}\end{array}$} & Platforms (KNO) & 3.98 & 1.88 & 4.29 & 1.86 & 4.22 & 1.97 & 0.96 & 0.30 & 0.23 & -0.06 \\
\hline & Blogs (KNO) & 4.02 & 1.75 & 4.29 & 1.72 & 4.51 & 1.82 & 1.86 & 0.27 & 0.48 & 0.21 \\
\hline & Wikis (KNO) & 3.49 & 1.91 & 3.95 & 1.92 & 4.58 & 1.90 & $7.48^{* *}$ & 0.46 & $10.09^{* *}$ & $0.63^{*}$ \\
\hline & Social networks (KN0) & 5.76 & 1.59 & 6.00 & 1.30 & 6.03 & 1.46 & 1.22 & 0.23 & 0.26 & 0.02 \\
\hline & Forums (KNO) & 5.04 & 1.61 & 5.37 & 1.35 & 5.55 & 1.72 & 2.94 & 0.32 & 0.50 & 0.17 \\
\hline & Chats (KNO) & 5.08 & 1.94 & 5.49 & 1.55 & 5.70 & 1.71 & $3.47^{*}$ & 0.40 & $0.62^{*}$ & 0.21 \\
\hline & Virtual tutorials (KNO) & 4.68 & 1.92 & 4.65 & 1.73 & 4.88 & 1.88 & 0.48 & -0.02 & 0.20 & 0.23 \\
\hline & Videoconferences (KNO) & 4.35 & 1.97 & 4.33 & 1.87 & 4.94 & 1.90 & $3.08^{*}$ & -0.02 & 0.58 & 0.60 \\
\hline & Video sharing (KNO) & 4.96 & 1.93 & 5.20 & 1.65 & 5.64 & 1.65 & $3.44^{*}$ & 0.23 & $0.67^{*}$ & 0.43 \\
\hline & Photo sharing (KN0) & 5.45 & 1.67 & 5.63 & 1.44 & 5.61 & 1.63 & 0.52 & 0.18 & 0.16 & -0.01 \\
\hline & Podcasts (KNO) & 3.84 & 2.01 & 3.92 & 2.00 & 4.18 & 2.08 & 0.68 & 0.08 & 0.34 & 0.25 \\
\hline & Social markers (KNO) & 4.36 & 2.12 & 4.94 & 1.70 & 5.41 & 1.87 & $7.40^{* *}$ & $0.58^{*}$ & $10.04^{* *}$ & 0.46 \\
\hline
\end{tabular}

The hypothesis was partially confirmed (4 out of 12 tools) along with the overall trend of the results. Students who make more use of the Internet are also more aware of Web 2.0 tools, especially wikis, chats, videoconferences, video sharing and social markers. They also know more about the rest of the tools (platforms, blogs, social networks, forums, virtual tutorials, photo sharing and podcasts), but the differences were not significant. 
- H7: Students who spend more time online use Web 2.0 tools more than students who spend fewer hours on the Internet.

Table 7. Internet hours and use of Web 2.0 tools $\left({ }^{*}=p<0.05 ;{ }^{* *}=p<0.01 ;{ }^{* * *}=p<0.001\right)$

\begin{tabular}{|c|c|c|c|c|c|c|c|c|c|c|c|}
\hline \multirow{3}{*}{$\frac{8}{8}$} & \multirow{3}{*}{ ITEM } & \multicolumn{6}{|c|}{ INTERNET HOURS } & \multirow{3}{*}{$\begin{array}{c}\text { ANOVA } \\
F\end{array}$} & \multicolumn{3}{|c|}{ MEAN DIFFERENCES } \\
\hline & & \multicolumn{2}{|c|}{$\begin{array}{c}1-2 h \text { (low) } \\
N=113\end{array}$} & \multicolumn{2}{|c|}{$\begin{array}{c}3-6 h \text { (medium) } \\
N=212\end{array}$} & \multicolumn{2}{|c|}{$\begin{array}{c}7 \text { or more h (high) } \\
\qquad N=78\end{array}$} & & \multirow{2}{*}{$\begin{array}{c}\text { Medium/ } \\
\text { low }\end{array}$} & \multirow{2}{*}{$\begin{array}{l}\text { High/ } \\
\text { low }\end{array}$} & \multirow{2}{*}{$\begin{array}{c}\text { High/ } \\
\text { medium }\end{array}$} \\
\hline & & $M$ & SD & $M$ & SD & $M$ & SD & & & & \\
\hline \multirow{12}{*}{$\frac{\frac{n}{3}}{\frac{3}{b}}$} & Platforms (USE) & 3.87 & 1.90 & 4.01 & 1.95 & 3.86 & 1.97 & 0.30 & 0.14 & -0.00 & -0.15 \\
\hline & Blogs (USE) & 3.26 & 1.87 & 3.50 & 1.74 & 3.94 & 1.98 & $3.15^{*}$ & 0.24 & $0.67^{*}$ & 0.43 \\
\hline & Wikis (USE) & 3.17 & 2.09 & 3.48 & 2.06 & 4.09 & 2.02 & $4.60^{*}$ & 0.31 & $0.92^{* *}$ & 0.61 \\
\hline & Social networking (USE) & 5.60 & 1.78 & 5.99 & 1.42 & 5.91 & 1.62 & 2.29 & 0.38 & 0.30 & -0.08 \\
\hline & Forums (USE) & 4.44 & 1.84 & 4.88 & 1.55 & 5.04 & 1.92 & $3.38^{*}$ & 0.43 & 0.59 & 0.16 \\
\hline & Chats (USE) & 4.41 & 2.16 & 5.00 & 1.80 & 4.92 & 2.08 & $3.37^{*}$ & $0.58^{*}$ & 0.51 & -0.07 \\
\hline & Virtual tutorials (USE) & 3.57 & 1.94 & 3.95 & 1.75 & 3.87 & 2.08 & 1.52 & 0.38 & 0.30 & -0.07 \\
\hline & Videoconferencing (USE) & 3.06 & 1.94 & 3.25 & 1.89 & 3.78 & 2.28 & $3.17^{*}$ & 0.18 & 0.72 & 0.53 \\
\hline & Video sharing (USE) & 4.16 & 2.11 & 4.86 & 1.54 & 5.12 & 1.86 & $8.14^{* * *}$ & $0.70^{* *}$ & $0.95^{* *}$ & 0.23 \\
\hline & Photo sharing (USE) & 4.92 & 1.88 & 5.45 & 1.48 & 5.09 & 2.01 & $3.88^{*}$ & $0.53^{*}$ & 0.17 & -0.36 \\
\hline & Podcasting (USE) & 3.04 & 1.85 & 3.37 & 1.98 & 3.55 & 2.19 & 1.72 & 0.33 & 0.51 & 0.18 \\
\hline & Social markers (USE) & 3.84 & 2.00 & 4.46 & 1.79 & 5.05 & 1.97 & $9.57^{* * *}$ & $0.61^{*}$ & $10.21^{* *}$ & 0.59 \\
\hline
\end{tabular}

The hypothesis was broadly confirmed (8 out of 12 tools). This trend was also confirmed in the rest of the tools. Students who spend more time online also tend to use more tools such as blogs, wikis, forums, chats, videoconferencing, video sharing and social markers. In addition, students who are medium-level Internet users are also more likely to use photo sharing tools. There were no significant differences in the use of distance training platforms, social networking, virtual tutorials or use of podcasts, but the trend was the same for these three latter tools.

\section{Discussion and conclusions}

Regarding the general hypothesis that students who spend more time on the Internet are positively differentiated from those who spend fewer hours in terms of their attitude, training, impact, perceived difficulties, knowledge and use of Web 2.0 in education, we may conclude that:

1. Major differences are observed in the factors: impact and use of web 2.0 tools, whereas minor differences are observed in the factors: attitude, training, difficulties and knowledge of Web 2.0 tools.

2. We found no differences, or very few, in attitude towards, training in, evaluation of use of and perception of difficulties regarding Web 2.0 integration in university education.

3. Students who spend more time on the Internet differ from those who spend fewer hours particularly in their consideration of Web 2.0 in education as lacking in teacher motivation, that the learning it provides is superficial, that it dehumanises teacher-student relations and that it encourages student individuality rather than socialisation. They are also more familiar with tools such as wikis, chats, videoconferencing, video sharing and social markers. Finally, they are also more assiduous users of tools like blogs, wikis, forums, chats, videoconferences, video sharing, photo sharing and social markers. 
4. In particular, students who spend fewer hours online differ from those that dedicate more time in their appraisal of Web 2.0 as an instrument for quality education. They also think that it helps personalise education, that students are familiar with it, and that it favours collaborative learning experiences, improves student-teacher communication, encourages the active role and commitment of students, improves course methodologies and generates more virtual tutorials. In general, they are aware of and use Web 2.0 to a lesser extent.

5. We may therefore say that overall the general hypothesis was partially confirmed.

In this sense, the results of our research are similar to those of other studies, such as Aguirre and Manasía (2009), Echeburúa and Corral (2010) and Ben and Dahmani (2008), which highlight the potential effects of Internet use in the medium and long term and the multitude of possibilities it provides. It is striking that students who spend more time using the Internet highlight the negative aspects of Web 2.0. Works such as those by Redecker et al. (2009) and Dabbagh and Reo (2011), analysing the impact of Web 2.0 in Higher Education institutions, show that the way the institution incorporates Web 2.0 into existing processes will determine the positive impact of this innovation.

In terms of the level of use and knowledge of tools, the outcomes are consistent with those of other studies, for example by Martínez et al. (2011), who reported a high level of awareness and use of the most popular Web 2.0 tools, including e-mail, the Internet and browsing, as well as basic use of IT applications.

On the other hand, students who spend less time online were more likely to emphasise the positive aspects of Web 2.0 use, although they are less familiar with the tools and tend to use them less. This coincides with the research carried out by Alba and Carballo (2005), in which the students perceived the advantages of Web 2.0 in day-to-day academic practice, although their knowledge and use of Web 2.0 tools was lower.

From the data analysed above, our interpretation is that university training in Web 2.0 may not be the result of institutional measures, but instead of personal interest and self-learning (Antón \& Zubillaga del Río, 2008).

To this end, it would be interesting to design training adapted to characteristics, needs and expectations, identifying preferences in the usefulness of Web 2.0.

The complexity of the subject calls for further research, taking into account other types of variables, such as age, gender and educational qualifications, which would allow the design of training plans to be tailored to the utility and features of the different degree courses.

\section{References}

Aguirre, A., \& Manasía, N. (2009). Web 2.0 y Web semántica en los entornos virtuales de aprendizaje. Multiciencias, 3(9), 320-328. Retrieved from http://www.redalyc.org/articulo.oa?id=90412325012

Alba, C. (2005). El profesorado y las tecnologías de la información y la comunicación en el proceso de convergencia al Espacio Europeo de Educación Superior. Revista de Educación, 337, 13-35. Retrieved from http://www.mecd. gob.es/revista-de-educacion/numeros-revista-educacion/numeros-anteriores/2005/re337/re337_02.html

Alba, C., \& Carballo, F. (2005). Viabilidad de las propuestas metodológicas para la aplicación del crédito europeo por parte del profesorado de las universidades españolas, vinculadas a la utilización de las TIC en la docencia y la investigación. Revista de Educación, 337, 71-97. Retrieved from http://www.mecd.gob.es/revista-de-educacion/ numeros-revista-educacion/numeros-anteriores/2005/re337/re337_05.html

RUSC VOL. 12 No 3 | Universitat Oberta de Catalunya and University of New England | Barcelona, July 2015

@Ángel Boza Carreño and Sara Conde Vélez @ @ by FUOC, 2015 | Relationship between hours spent on the Internet and Web 2.0 in Higher Education 
Albirini, A. (2006). Teachers' attitudes toward information and communication technologies: the case of Syrian EFL teachers. Computers \& Education, 47, 373-398. doi: http://dx.doi.org/10.1016/j.compedu.2004.10.013

Antón, P., \& Zubillaga del Río, A. (2008). La formación del profesorado para la implantación de las TIC como soporte a los nuevos modelos derivados del Espacio Europeo de Educación Superior (EEES). Madrid, Spain: Universidad Complutense de Madrid. Retrieved from http://es.scribd.com/doc/245777583/Formacion-Prof-Tics-Eees

Area, M. (2004). Las nuevas tecnologías de la información y la comunicación en el contexto de la educación superior. Los medios y las tecnologías en la educación. Madrid, Spain: Pirámide.

Ballesteros, C., Cabero, J., Llorente M. C., \& Morales, J. A. (2010). Usos del e-learning en las universidades andaluzas. Estado de la situación y análisis de buenas prácticas. Pixel-Bit. Revista de Medios y Educación, 37, 7-18. Retrieved from http://tecnologiaedu.us.es/tecnoedu/images/stories/jca47.pdf

Ben, A., \& Dahmani, M. (2008). The Impact of ICT on Student Performance in Higher Education: Direct Effects, Indirect Effects and Organizational Change. RUSC. Universities and Knowledge Society Journal, 1(5). Retrieved from http://www.uoc.edu/rusc/5/1/dt/eng/benyoussef_dahmani.pdf

Boza, Á., \& Conde, S. (2015). Training, attitude, use and impact of Web 2.0 in higher education: scale validation / Formación, actitud, uso e impacto de la web 2.0 en educación superior: validación de una escala. Cultura y Educación. http://www.tandfonline.com/doi/full/10.1080/11356405.2015.1034531

Boza, Á., Tirado, R., \& Guzmán, M. D. (2010). Creencias del profesorado sobre el significado de la tecnología en la enseñanza: influencia para su inserción en los centros docentes andaluces. RELIEVE, 1(16), 1-24. Retrieved from http://www.uv.es/RELIEVE/v16n1/RELIEVEV16n1_5.htm

British Educational Communications and Technology Agency (BECTA). (2004). A Review of the Research Literature on Barriers to the Uptake of ICT by Teachers. London, United Kingdom: BECTA. Retrieved from http://partners.becta. org.uk/page_documents/research/barriers.pdf

Cabero, J. (2014). Formación del profesorado universitario en TIC. Aplicación del método Delphi para la selección de los contenidos formativos. Educación XX1, 17(1), 111-132. Retrieved from http://e-spacio.uned.es/fez/eserv. php?pid=bibliuned:EducacionXXI-2014-17-1-7040\&dsID=Documento.pdf

Casas, J. A., Ruiz-Olivares, R., \& Ortega-Ruiz, R. (2013). Validation of the Internet and Social Networking Experiences Questionnaire in Spanish adolescents. International Journal of clinical and Health psychology, 13, 40-48. doi: http://dx.doi.org/10.1016/S1697-2600(13)70006-1

Cavas, B., Cavas, P., Karaoglan, B., \& Kisla, T. (2009). A study on science teachers' attitudes toward information and communication technologies in education. The Turkish Online Journal of Educational Technology, Tojet. Retrieved from http://www.tojet.net/articles/v8i2/822.pdf

Cuadrado, I. (2008). Estado de necesidades, desarrollo y evaluación en Formación del Profesorado en TICs: Extremadura. Avances en Supervisión Educativa, 8, 1-13. Retrieved from http://www.adide.org/revista/index. php?option=com_content\&task=view\&id=442\&ltemid=64

Dabbagh, N., \& Reo, R. (2011). Impact of Web 2.0 on Higher Education. In D. W. Surry, R. M. Gray, \& J. R. Stefurak (2011). Technology Integration in Higher Education: Social and Organizational Aspects (pp. 174-187). Hershey, PA: IGI Global. doi: http://dx.doi.org/10.4018/978-1-60960-147-8.ch013

Díez, E. (2012). Modelos socioconstructivistas y colaborativos en el uso de las TIC en la formación inicial del profesorado. Revista de Educación, 358, 175-196. Retrieved from http://www.mecd.gob.es/revista-de-educacion/ numeros-revista-educacion/numeros-anteriores/2012/re358/re358_09.html

RUSC VOL. 12 No 3 | Universitat Oberta de Catalunya and University of New England | Barcelona, July 2015

@ Ángel Boza Carreño and Sara Conde Vélez | @ by FUOC, 2015 | Relationship between hours spent on the Internet and Web 2.0 in Higher Education 
Echeburúa, E., \& Corral, P. (2010). Adicción a las nuevas tecnologías y a las redes sociales en jóvenes: un nuevo reto. ADICCIONES, 2(22), 91-96. Retrieved from http://www.adicciones.es/files/91-96\%20editorial\%20echeburua.pdf Ertmer, P. (2005). Teacher pedagogical beliefs: The final frontier in our quest for technology integration. Educational Technology, Research and Development, 53(4), 25-39. doi: http://dx.doi.org/10.1007/BF02504683

Garay, U., Luján, C., \& Etxebarría, A. (2013). El empleo de herramientas de la web 2.0 para el desarrollo de estrategias cognitivas: un estudio comparativo. Porta Linguarum, 20, 169-186. Retrieved from http://www.ugr.es/ portalin/ articulos/PL_numero20/11\%20\%20Urtza.pdf

Hinojo, F. J., \& Fernández, F. (2002). Diseño de escalas de actitudes para la formación del profesorado en tecnologías. Comunicar, 19, 120-125. Retrieved from http://www.revistacomunicar.com/index.php?contenido=detalles\&nu mero=19\&articulo=19-2002-21

Martínez, J., Amenabar, N., \& Lareki, A. (2011). Preferencias del profesorado universitario sobre modalidades formativas en TIC. Revista de Psicodidáctica, 16(1), 85-98. Retrieved from www.ehu.es/revista-psicodidactica

Osborne, J., \& Hennessy, S. (2003). Literature review in science education and the role of ICT: Promise, problems and future directions. London, United Kingdom: Futurelab.

Pérez Ríos, J. (2003). ¿Cómo usa el profesorado las nuevas tecnologías? Granada, Spain: Ed. Universitario.

Redecker, C., Ala-Mutka, K., Bacigahpo, M., Ferrari, A., \& Punie, Y. (2009). Learning 2.0: The Impact of Web 2.0 Innovations on Education and Training in Europe. JRC European Commission.

Ruiz, I., Rubia, B., Martínez. R., \& Fernández. E. (2010). Formar al profesorado inicialmente en habilidades y competencias en TIC: perfiles de una experiencia colaborativa. Revista de Educación, 352, 149-178. Retrieved from http://www.mecd.gob.es/dctm/revista-de-educacion/articulosre352/re35207.pdf?documentld $=0901 \mathrm{e} 72 \mathrm{~b} 812342 \mathrm{c} 4$

Sánchez, M., \& Mayor, C. (2006). Los jóvenes profesores universitarios y su formación pedagógica. Claves y controversias. Revista de Educación, 339, 923-946. Retrieved from http://www.mecd.gob.es/revista-de-educacion/ numeros-revista-educacion/numeros-anteriores/2006/re339/re339_39.html

Sangrà, A., \& González Sanmamed, M. (Eds.). (2004). La transformación de las universidades a través de las TIC: discursos y prácticas. Barcelona, Spain: Editorial UOC.

Suárez, C. (2009). Estructura didáctica virtual para Moodle. Didáctica, Innovación y Multimedia, 13. Retrieved from http://www.pangea.org/dim/revistaDIM13/Articulos/cristobalsuarez.doc

Tejedor, F. J., García, A., \& Prada, S. (2009). Medidas de actitudes del profesorado universitario hacia la integración de las TIC. Comunicar, 33, 115-124. doi: http://dx.doi.org/10.3916/c33-2009-03-002

Toprakci, E. (2006). Obstacles at integration of schools into information and communication technologies by taking into consideration the opinions of the teachers and principals of primary and secondary schools in Turkey. Journal of Instructional Science and Technology (e-JIST), 9(1), 1-16.

Valdés, A., Angulo, J., Urías, M., García, R., \& Mortis, V. (2011). Necesidades de capacitación de docentes de educación básica en el uso de las TIC. Pixel-Bit. Revista de Mediosy Educación, 39, 211-223. Retrieved from http://www.redalyc. org/pdf/368/36818685016.pdf

RUSC VOL. 12 No 3 | Universitat Oberta de Catalunya and University of New England | Barcelona, July 2015 


\section{About the authors}

\section{Ángel Boza Carreño}

aboza@uhu.es

ORCID ID: http://orcid.org/0000-0002-3395-421X

Doctor of Educational Psychology, University of Huelva (UHU), Spain

He holds a bachelor's degree in Education from the National University of Distance Education (UNED), Spain. He is a lecturer in the Education Department and director of the master's degree programme in Educational Guidance at the University of Huelva (UHU), Spain. His lines of research are educational guidance, ICTs and education, and academic motivation.

Universidad de Huelva

Departamento de Educación, Facultad de Ciencias de la Educación

Campus de El Carmen

Avda. Tres de Marzo, s/n

21007 Huelva

Spain

Sara Conde Vélez

sara.conde@dedu.uhu.es

ORCID ID: http://orcid.org/0000-0002-7950-5866

Doctor in Educational Psychology, University of Huelva (UHU), Spain

She is a lecturer in Research and Diagnosis Methods in Education (MIDE). She studies coexistence in schools and has had several works published on her lines of research into school coexistence and the role of social educators in managing coexistence.

Universidad de Huelva

Departamento de Educación, Facultad de Ciencias de la Educación

Campus de El Carmen

Avda. Tres de Marzo, s/n

21007 Huelva

Spain

Original title: Relación entre horas dedicadas a internet y web 2.0 en educación universitaria

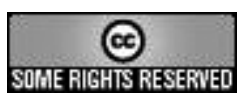

The texts published in this journal are - unless indicated otherwise - covered by the Creative Commons Spain Attribution 3.0 licence. You may copy, distribute, transmit and adapt the work, provided you attribute it (authorship, journal name, publisher) in the manner specified by the author(s) or licensor(s). The full text of the licence can be consulted here: <http://creativecommons.org/licenses/by/3.0/es/deed.en>

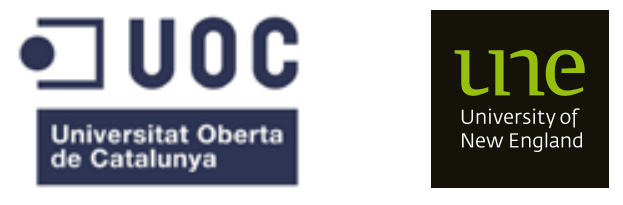

